\title{
Radio Mean Labeling Of Paths And Its Total Graph
}

\author{
${ }^{1}$ Meera Saraswathi, ${ }^{2}$ K. N. Meera \\ 1Dept. of Mathematics, Amrita School of Arts and Sciences, Kochi \\ Affiliated to Amrita Vishwa Vidyapeetham \\ India \\ 2Dept. of Mathematics, Amrita School of Engineering, Bengaluru \\ Affiliated to Amrita Vishwa Vidyapeetham \\ India \\ Email: kn_meera@blr.amrita.edu
}

Article History: Received: 11 January 2021; Accepted: 27 February 2021; Published online: 5 April 2021

\begin{abstract}
A graph labeling problem is an assignment of labels to the vertices or edges (or both) of a graph $G$ satisfying some mathematical condition. Radio Mean Labeling, a vertex-labeling of graphs with non-negative integers has a significant application in the study of problems related to radio channel assignment. The maximum label used in a radio mean labeling is called its span, and the lowest possible span of a radio mean labeling is called the radio mean number of a graph. In this paper, we obtain the radio mean number of paths and total graph of paths.
\end{abstract}

Keywords - Channel assignment problem; Graph theory; Path graph; Radio Mean Labeling; Total graph of a graph

\section{INTRODUCTION}

For basic graph theory terminology, we refer [16]. The basic principle of a Radio communication network is transmission and reception of radio signals. Each radio station is assigned a channel number or frequency; transmitter sends signals; a receiver then picks it up and translates it to the sounds heard through the radio. However, the reception will be degraded by the unnecessary interference by transmitters of closely related channel number, if any. Hence the channel assignment problem is to assign radio channels to transmitters with minimum span in such a way that it minimizes interference between radio stations that are in the same neighborhood. This problem of Radio channel assignment can be converted into a Graph theoretic problem as follows: The radio network can be considered as a graph in which vertices corresponds to transmitter locations and two vertices are adjacent if the locations of the radio stations corresponding to these vertices are close. The main objective is to label vertices of this graph with minimum span where the labels given to the vertices determine the channel on which it transmits [15]. Chartrand et al. converted this problem to a vertex labeling problem as follows: For a connected graph $G$, radio labeling was defined as a one-to-one function $\varphi$ from $V(G)$ to $\mathbb{Z}^{+}$, the set of all positive integers where $d(u, v)+|\varphi(u)-\varphi(v)| \geq 1+\operatorname{diam}(G), \forall u, v \in V(G)$. Authors in [17] studied the Radio labeling of Strong product of $\mathrm{K}_{3}$ and $\mathrm{P}_{\mathrm{n}}$. Graphs for which the largest label used is same as the order of the graph are called radio graceful. In [10], [11] the authors study this concept of radio gracefulness of a graph.

The idea of radio mean labeling of graphs was conceived the paper [5], published in the year 2015. The radio mean labeling of a connected graph $G$ was defined as an injective function

$$
\begin{aligned}
& f: V(G) \rightarrow \mathbb{Z}^{+} \text {where } \\
& \left\lceil\frac{f(u)+f(v)}{2}\right\rceil+d(u, v) \geq \operatorname{diam}(G)+1, \forall u, v \in V(G) .
\end{aligned}
$$

The radio mean number of $f$ or $r m n(f)$ is the maximum integer assigned to any $v \in V(G)$ under this mapping $f$. Further the radio mean number of $G$, denoted by $\operatorname{rmn}(G)$ is the smallest value of $r m n(f)$ taken over all radio mean labelings $f$ of $G$. It is obvious by the definition that $\operatorname{rmn}(G) \geq|V(G)|$. If $\operatorname{rmn}(G)=$

$|V(G)|$, then $G$ is called a radio mean graceful graph [3].

In [5, 6, 7, 8, 9], Ponraj, R., S. Sathish Narayanan and R. Kala have investigated the radio mean labeling of many classes of graphs including graphs with maximum distance between distinct pairs of vertices either two or three. The radio mean number of Triangular Ladder graph, corona $\mathrm{P}_{\mathrm{n}}$ with $\square \mathrm{K}_{2}$, corona $\mathrm{K}_{\mathrm{n}}$ with $\square \mathrm{K}_{2}$ and corona $\mathrm{W}_{\mathrm{n}}$ with $\square \mathrm{K}_{2}$ are obtained in [13] by Sunitha, K., C. David Raj and A. Subramanian and that of subdivision graph of complete graphs, Mongolian tent graph, subdivision of Friendship graphs, and diamond graphs in [10] by Lavanya Y. and K. N. Meera. Smitha, KM Baby and K. Thirusangu studied the radio mean labeling of corona $K_{m}$ with $K_{n}$, corona $W_{m}$ with $K_{n}^{-}$, corona star $S_{m}$ with $K_{n}{ }^{-}$and corona Helm $\mathrm{H}_{m}$ with $K_{n}{ }^{-}$in [12]. In [11], Raj, Deva and Brindha studied the radio mean labeling of Degree Splitting graph of $P_{n}, K_{1, n}$ and corona $P_{n}$ with $\mathrm{K}_{1}$. In [4] the mean in the definition is replaced by geometric mean and radio geometric graceful graphs are studied. 
The total graph of a graph $G$ is a graph whose vertex set is $V(G) \cup E(G)$, and two vertices are adjacent in the total graph if and only if they are adjacent or incident in $G$. We denote total graph of $G$ by $T(G)$.

\section{RADIO MEAN LABELING OF PATHS}

Consider the Path $P_{n}$ on $n$ vertices $v_{l}, v_{2}, \cdots, v_{n}$. Note that for path graph $P_{n}$, $\operatorname{diam}\left(P_{n}\right)=\mathrm{n}-1$. A labeling $f: V\left(P_{n}\right) \rightarrow \mathbb{Z}^{+}$is a Radio mean labeling of $\mathrm{P}_{\mathrm{n}}$, if $f$ is injective and satisfies the condition:

$$
\left\lceil\frac{f\left(v_{i}\right)+f\left(v_{j}\right)}{2}\right\rceil+d\left(v_{i}, v_{j}\right) \geq n, \text { for all } v_{i}, v_{j} \in V\left(P_{n}\right) \text {. (1) }
$$

A. Case I: $n=2,3$

Define $f: V\left(P_{n}\right) \rightarrow \mathbb{Z}^{+}$defined by $f\left(v_{i}\right)=i$, where $v_{i} \in V\left(P_{n}\right)$ for each $\mathrm{n} \in\{2,3\}$. It can easily be seen that paths $P_{2}, P_{3}$ admit Radio mean Graceful labeling under the injective mapping $f$.

B. Case II: $n \geq 4$

\section{Theorem: II-B.I}

The Path $P_{n}, n=4,5,6$ admits Radio mean labeling with $r m n\left(P_{n}\right)=2 n-4$.

Proof. For $\mathrm{n}=4,5,6$, define a function $f: V\left(P_{n}\right) \rightarrow \mathbb{Z}^{+}$by $f\left(v_{1}\right)=n-3, f\left(v_{2}\right)=2 n-4, f\left(v_{3}\right)=n-2, f\left(v_{i}\right)=2 n$ $-i-1: 4 \leq i \leq n$. Clearly, $f$ is an injective function. We shall now show that the function $f$ satisfies (1). Consider any pair $\left(v_{i}, v_{j}\right) \in V\left(P_{n}\right)$.

$$
\begin{aligned}
\left\lceil\frac{f\left(v_{i}\right)+f\left(v_{j}\right)}{2}\right\rceil+d\left(v_{i}, v_{j}\right) & \geq\left\lceil\frac{f\left(v_{1}\right)+f\left(v_{3}\right)}{2}\right\rceil+d\left(v_{1}, v_{3}\right) \\
& \geq\left\lceil\frac{n-3+n-2}{2}\right\rceil+2 \\
& \geq n-2+2 \\
& \geq n
\end{aligned}
$$

Under this labeling $f$, every pair of vertices in $P_{n}, \mathrm{n}=4,5,6$ satisfies radio mean condition and hence $f$ is a radio mean labeling of $P_{n}$. The maximum integer used as a label under this function $f$ is $2 \mathrm{n}-4$ and so $r m n(f)=$ $2 \mathrm{n}-4$. When $\mathrm{n}=4, f$ is a graceful labeling and so $r m n\left(P_{n}\right)=2 \mathrm{n}-4=\mathrm{n}$ and for $\mathrm{n}=5,6, \operatorname{rmn}\left(P_{n}\right) \leq 2 \mathrm{n}-4$.

It is clear from the definition of $f$ that any radio mean labeling of $P_{n}, \mathrm{n}=5,6$ whose range consists of only integers greater than $\mathrm{n}-3$ has a span greater than that of $f$. Let us now consider any radio mean labeling $h$ of $P_{n}$ , $\mathrm{n}=5,6, h: V\left(P_{n}\right) \rightarrow\{n-4, n-3, n-2, \cdots\}$. Then it follows from the Radio mean condition that any vertices receiving labels $n-4$ and $n-3$ are at least $n-2$ distance apart, any vertices receiving labels $n-3$ and $n-2$ are at least $\mathrm{n}-3$ distance apart and any vertices receiving labels $\mathrm{n}-4$ and $\mathrm{n}-2$ are at least $\mathrm{n}-2$ distance apart. A labeling of $P_{n}$ using integers $\{\mathrm{n}-4, \mathrm{n}-3, \mathrm{n}-2\}$ satisfying the above constraints on distance is not feasible. This indicates that not all of the integers $\{\mathrm{n}-4, \mathrm{n}-3, \mathrm{n}-2\}$ are in the Range of $h$ and so span of $h$ is greater than $2 \mathrm{n}-5$. In other words, $r m n(h) \geq 2 \mathrm{n}-4$. Thus, we can show that any radio mean labeling of $P_{n}, \mathrm{n}=5,6$ whose range set consists of integers less than $\mathrm{n}-3$ has span greater than or equal to $2 \mathrm{n}-4$. Hence, $\operatorname{rmn}\left(P_{n}\right)=2 \mathrm{n}-4, \mathrm{n}$ $=5,6$. Hence, for path $P_{n}, \mathrm{n}=4,5,6$ we have $\operatorname{rmn}\left(P_{n}\right)=2 \mathrm{n}-4$.

\section{Lemma: II-B.1}

Suppose $n$ is any integer which belongs to an interval of the form : $\left[4+S_{k}, 6+S_{k}+k\right]$ where $S_{k}=3+4+5+\cdot$. $+(3+k-1)$ and $k=1,2,3, \cdots$. Then there exists a Radio mean labeling of $P_{n}, n \geq 7$ with radio mean number, $\operatorname{rmn}\left(P_{n}\right)=2 n-k-4$.

Proof. Suppose $n \in\left[4+S_{k}, 6+S_{k}+k\right]$ where $S_{k}=3+4+5+\cdots+(3+k-1)$ and $k=1,2,3, \cdots$. Let us define a function $f$,

$f: V\left(P_{n}\right) \longrightarrow \mathbb{Z}^{+}$using indices $d_{1}, d_{2}, \cdots, d_{k+2}$ where $d_{1}=$

1 , and $d_{i}=d_{i-1}+n-f\left(v_{d_{i-1}}\right)-1 ; i=2,3, \cdots, k+2$.

The function $f$ is given by

$$
\begin{aligned}
\text { (i) } \quad f\left(v_{1}\right)=f\left(v_{d_{1}}\right)=n-k-3 \\
\text { (ii) } \quad f\left(v_{d_{i}}\right)=f\left(v_{d_{1}}\right)+i-1=n-k+i-4, \\
\text { if } i=2,3, \cdots, k+2 \\
\text { (iii) } \quad f\left(v_{i}\right)=\quad f\left(v_{d_{k+2}}\right)+n-i+1=2 n-i-1, \\
\text { if }\left(d_{k+2}\right)+1 \leq i \leq n \\
\text { (iv) } \quad f\left(v_{i}\right)=\quad f\left(v_{\left(d_{l}+1\right)}\right)+d_{l}-i, \text { if } i \in\left(d_{l-1}, d_{l}\right) \\
\text { where } l \in\{k+2, k+1, k, \cdots, 3,2\}
\end{aligned}
$$

Clearly, $f$ is an injective function. We shall now show that the $f$ satisfies (1). Following are the different cases we consider: 
(1) Consider the pair $\left(v_{i}, v_{j}\right)$ where $v_{i}, v_{j} \in$ $\left\{v_{d_{1}}, v_{d_{2}}, \cdots, v_{d_{k+2}}\right\}$

$$
\begin{aligned}
\left\lceil\frac{f\left(v_{i}\right)+f\left(v_{j}\right)}{2}\right\rceil+d\left(v_{i}, v_{j}\right) \geq & \left\lceil\frac{f\left(v_{d_{1}}\right)+f\left(v_{d_{2}}\right)}{2}\right\rceil \\
& +d\left(v_{d_{1}}, v_{d_{2}}\right) \\
\geq & f\left(v_{d_{1}}\right)+1+d_{2}-1 \\
\geq & n
\end{aligned}
$$

(2) Consider the pair $\left(v_{i}, v_{j}\right)$ where $\left(d_{k+2}\right)+1 \leq i$ and $j \leq n, i \neq j$.

$$
\begin{aligned}
\left\lceil\frac{f\left(v_{i}\right)+f\left(v_{j}\right)}{2}\right\rceil+d\left(v_{i}, v_{j}\right) \geq & 2 n-1+\frac{i+j}{2}+ \\
& d\left(v_{i}, v_{j}\right) \\
\geq & n
\end{aligned}
$$

(3) Consider the pair $\left(v_{i}, v_{j}\right)$ where $i \in\left\{d_{1}, d_{2}, \cdots, d_{k+2}\right\}$ and $\left(d_{k+2}\right)+1 \leq j \leq n$.

$$
\begin{aligned}
\left\lceil\frac{f\left(v_{i}\right)+f\left(v_{j}\right)}{2}\right\rceil+d\left(v_{i}, v_{j}\right) & \geq \frac{3}{2} n+\frac{j}{2}-\frac{k}{2}-3 \\
& \geq n
\end{aligned}
$$

(4) Consider the pair $\left(v_{i}, v_{j}\right)$ where $v_{i}, v_{j} \in V\left(P_{n}\right)$

$$
\begin{aligned}
&\left(\left\{v_{d_{1}}, v_{d_{2}}, \cdots, v_{d_{k+2}}\right\} \bigcup\left\{v_{\left(d_{k+2}\right)+1}, v_{\left(d_{k+2}\right)+2}, \cdots, v_{n}\right\}\right) \\
&\left\lceil\frac{f\left(v_{i}\right)+f\left(v_{j}\right)}{2}\right\rceil+d\left(v_{i}, v_{j}\right) \geq f\left(v_{d_{k+2}-1}\right)+d\left(v_{i}, v_{j}\right) \\
& \geq 2 n-d_{k+2}-2 \\
&+d\left(v_{i}, v_{j}\right) \\
& \geq n
\end{aligned}
$$

(5) Consider the pair $\left(v_{i}, v_{j}\right)$ where $v_{i} \in\left\{v_{d_{1}}, \cdots, v_{d_{k+2}}\right\}$ and

$$
\begin{aligned}
& v_{j} \in V\left(P_{n}\right) \backslash\left(\left\{v_{d_{1}}, \cdots, v_{d_{k+2}}\right\} \bigcup\left\{v_{\left(d_{k+2}\right)+1}, \cdots, v_{n}\right\}\right) \\
&\left\lceil\frac{f\left(v_{i}\right)+f\left(v_{j}\right)}{2}\right\rceil+d\left(v_{i}, v_{j}\right) \geq\left\lceil\frac{n-3+2 n-d_{k+2}}{2}\right\rceil \\
&+1 \\
& \geq\left\lceil\frac{\left.3 n-d_{k+2}-3\right)}{2}\right\rceil+1 \\
& \geq n
\end{aligned}
$$

(6) Consider the pair $\left(v_{i}, v_{j}\right)$ where

$$
\begin{aligned}
& v_{i} \in\left\{v_{\left(d_{k+2}\right)+1}, v_{\left(d_{k+2}\right)+2}, \cdots, v_{n}\right\} \text { and } \\
& v_{j} \in V\left(P_{n}\right) \backslash\left(\left\{v_{d_{1}}, \cdots, v_{d_{k+2}}\right\} \bigcup\left\{v_{\left(d_{k+2}\right)+1}, \cdots, v_{n}\right\}\right) \\
& \left.\qquad \frac{f\left(v_{i}\right)+f\left(v_{j}\right)}{2}\right\rceil+d\left(v_{i}, v_{j}\right) \geq \\
& \left.\geq \frac{2 f\left(v_{\left(d_{k+2}\right)+1}\right)+1}{2}\right\rceil \\
& \geq \\
& \geq f\left(v_{\left(d_{k+2}\right)+1}\right)+3 \\
& \geq 2 n-\left(d_{k+2}+1\right)+2 \\
& \geq n
\end{aligned}
$$

From all the above cases it follows that $f$ satisfies Radio mean condition for all pairs of vertices of $P_{n}, \mathrm{n} \geq 7$. The maximum number assigned to any vertex of $P_{n}$ under this mapping is $2 \mathrm{n}-\mathrm{k}-4$. Hence the radio mean number of $f, r m n(f)=2 \mathrm{n}-\mathrm{k}-4$.

\section{Theorem: II-B.2}

For any Path $P_{n}, n \in\left[4+S_{k}, 6+S_{k}+k\right]$ where $S_{k}=3+4+5+\cdots+(3+k-1)$ and $k=1,2,3, \cdots$ as in previous lemma the radio mean number of $P_{n}$ is $2 n-k-4$. 
Proof. It is clear from the function $f$ defined in previous lemma that any radio mean labeling of $P_{n}$ whose range contains only integers greater than $\mathrm{n}-\mathrm{k}-3$ has a span greater than that of $f$. Now we shall investigate about the span of radio mean labelings of $P_{n}$ whose range consists of integers less than $\mathrm{n}-\mathrm{k}-3$. It is observed that under the labeling $f$,

$$
\begin{aligned}
& d\left(v_{d_{1}}, v_{d_{2}}\right)+d\left(v_{d_{2}}, v_{d_{3}}\right)+\cdots+d\left(v_{d_{k+1}}, v_{d_{k+2}}\right)+1 \leq \operatorname{diam}\left(P_{n}\right) \\
& \text { i.e }(k+2)+(k+1)+k+\cdots+2+1=4+S_{k}-1 \leq n-1
\end{aligned}
$$

Consider any radio mean labeling $h$ of $P_{n}, h: V\left(P_{n}\right) \rightarrow\{n-k-4, n-k-3, n-k-2, \cdots\}$. Then it follows from Radio mean condition that any vertices receiving labels $n-k-4$ and $n-k-3$ must be at least $k+3$ distance apart, any vertices receiving labels $\mathrm{n}-\mathrm{k}-3$ and $\mathrm{n}-\mathrm{k}-2$ must be at least $\mathrm{k}+2$ distance apart, any vertices receiving labels $\mathrm{n}-\mathrm{k}-2$ and $\mathrm{n}-\mathrm{k}-1$ must be at least $\mathrm{k}+1$ distance apart, $\cdots$, any vertices receiving labels $(\mathrm{n}-\mathrm{k}-3)+\mathrm{k}$ and $(\mathrm{n}-\mathrm{k}-3)+(\mathrm{k}+1)$ must be at least 2 distance apart. If $\{(\mathrm{n}-\mathrm{k}-4),(\mathrm{n}-\mathrm{k}-3),(\mathrm{n}-\mathrm{k}$ $-2), \cdots,(\mathrm{n}-\mathrm{k}-3)+\mathrm{k},(\mathrm{n}-\mathrm{k}-3)+(\mathrm{k}+1)\}$ are in the Range of $\mathrm{h}$, then we must have

$$
(\mathrm{k}+3)+(\mathrm{k}+2)+(\mathrm{k}+1) \cdots+3+2+1 \leq \mathrm{n}-1
$$

That implies $(\mathrm{k}+3)+4+S_{k}-1 \leq \mathrm{n}-1$, a contradiction since $4+\mathrm{S}_{\mathrm{k}} \leq \mathrm{n} \leq 6+\mathrm{S}_{\mathrm{k}}+\mathrm{k}$. This means not all of the integers

Fig:1 Path on 8 vertices

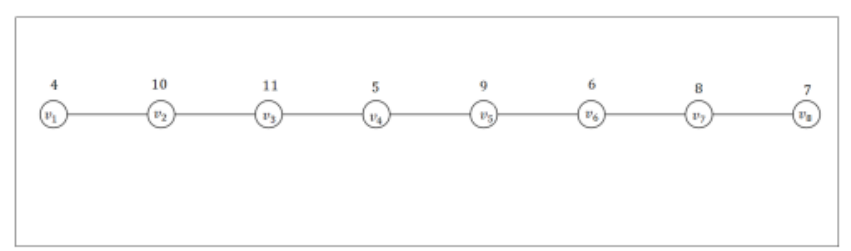

$\{(\mathrm{n}-\mathrm{k}-4),(\mathrm{n}-\mathrm{k}-3),(\mathrm{n}-\mathrm{k}-2), \cdots,(\mathrm{n}-\mathrm{k}-3)+\mathrm{k},(\mathrm{n}-\mathrm{k}-3)+(\mathrm{k}+1)\}$ are in the Range of $h$ which implies that the maximum integer assigned to any vertex of $P_{n}$ under the mapping $h, r m n(h)>2 \mathrm{n}-\mathrm{k}-5$. In other words, $r m n(h) \geq 2 \mathrm{n}-\mathrm{k}-4$.

Similarly, we can show that any radio mean labeling of $P_{n}$ whose range set includes integers less than $\mathrm{n}-\mathrm{k}-$ 3 has span greater than or equal to $2 \mathrm{n}-\mathrm{k}-4$. Therefore, for path $P_{n}, 4+\mathrm{S}_{\mathrm{k}} \leq \mathrm{n} \leq 6+\mathrm{S}_{\mathrm{k}}+\mathrm{k}, \operatorname{rmn}\left(P_{n}\right)=2 \mathrm{n}-\mathrm{k}-$ 4.

\section{RADIO MEAN LABELING OF TOTAL GRAPH OF PATHS}

The total graph of a path $P_{n}$ is a graph whose vertex set consists of the vertices and edges of $P_{n}$ and two vertices are adjacent in $T\left(P_{n}\right)$ if and only if their corresponding elements are either adjacent or incident in $P_{n}$. Let $P_{n}$ be a path of $\mathrm{n}$ vertices namely $v_{1}, v_{2}, \cdots, v_{n}$ and edges $e_{1}, e_{2}, \cdots, e_{n}$. Then the total graph of $P_{n}$ denoted by $T\left(P_{n}\right)$ is the graph with vertex set

$$
\left\{v_{1}, v_{2}, \cdots, v_{n}\right\} \cup\left\{v_{1}^{\prime}, v_{2}^{\prime}, \cdots, v_{n-1}^{\prime}\right\}
$$

Where $v_{i}^{\prime}$ is the vertex corresponding to edge $e_{i}$ of $P_{n}$ and two vertices of $T\left(P_{n}\right)$ are adjacent if their corresponding elements are adjacent in $P_{n}$. It is obvious that the diameter of $T\left(P_{n}\right)$ is equal to $\mathrm{n}-1$. A labeling $f: V\left(T\left(P_{n}\right)\right) \rightarrow \mathbb{Z}^{+}$is a radio mean labeling of $T\left(P_{n}\right)$, if $T\left(P_{n}\right)$ is injective and satisfies the condition

$$
\left\lceil\frac{f(u)+f(v)}{2}\right\rceil+d(u, v) \geq n, \text { for all } u, v \in V\left(\mathcal{T}\left(P_{n}\right)\right) .
$$

\section{A. Case I: $n=2,3,4$}

\section{Theorem: III-A.I}

The total graph $T\left(P_{n}\right)$ of Path $P_{n}, n=2,3,4$ admits Radio mean graceful labeling.

Proof. Define $f: V\left(T\left(P_{n}\right)\right) \rightarrow \mathbb{Z}^{+}$as follows.

$$
\begin{aligned}
& f\left(v_{1}\right)=2, f\left(v_{n}\right)=1, \\
& f\left(v_{i}\right)=2+2(i-1): 2 \leq i<n, \\
& f\left(v_{i}^{\prime}\right)=3+2(i-1): 1 \leq i \leq n-1
\end{aligned}
$$

Clearly, $f$ is an injective function. We shall now show that the f satisfies (2). Following are the different cases we consider: 
(1) Consider the pair $\left(v_{i}, v_{j}^{\prime}\right)$ where $v_{i}, v_{j}^{\prime} \in V\left(\mathcal{T}\left(P_{2}\right)\right)$

$$
\begin{aligned}
\left\lceil\frac{f\left(v_{i}\right)+f\left(v_{j}^{\prime}\right)}{2}\right\rceil+d\left(v_{i}, v_{j}^{\prime}\right) \geq & \left\lceil\frac{f\left(v_{1}\right)+f\left(v_{2}\right)}{2}\right\rceil+ \\
& d\left(v_{1}, v_{2}\right) \\
\geq & \left\lceil\frac{1+2}{2}\right\rceil+1=3 \\
& >2
\end{aligned}
$$

(2) If $v_{i}, v_{j} \in V\left(\mathcal{T}\left(P_{n}\right)\right), n=3,4$

$$
\begin{aligned}
\left\lceil\frac{f\left(v_{i}\right)+f\left(v_{j}\right)}{2}\right\rceil+d\left(v_{i}, v_{j}\right) \geq & \left\lceil\frac{f\left(v_{1}\right)+f\left(v_{n}\right)}{2}\right\rceil+ \\
& d\left(v_{1}, v_{n}\right) \\
\geq & \left\lceil\frac{2+1}{2}\right\rceil+n-1 \\
\geq & 2+n-1 \\
> & n
\end{aligned}
$$

(3) If $v_{i}^{\prime}, v_{j}^{\prime} \in V\left(\mathcal{T}\left(P_{n}\right)\right), n=3,4$

$$
\begin{aligned}
\left\lceil\frac{f\left(v_{i}^{\prime}\right)+f\left(v_{j}^{\prime}\right)}{2}\right\rceil+d\left(v_{i}^{\prime}, v_{j}^{\prime}\right) \geq & \left\lceil\frac{f\left(v_{1}^{\prime}\right)+f\left(v_{2}^{\prime}\right)}{2}\right\rceil+ \\
& d\left(v_{1}^{\prime}, v_{2}^{\prime}\right) \\
\geq & \left\lceil\frac{3+5}{2}\right\rceil+1=5 \\
&
\end{aligned}
$$

(4) If $v_{i}, v_{j}^{\prime} \in V\left(\mathcal{T}\left(P_{n}\right)\right), n=3,4$

$$
\begin{aligned}
\left\lceil\frac{f\left(v_{i}\right)+f\left(v_{j}^{\prime}\right)}{2}\right\rceil+d\left(v_{i}, v_{j}^{\prime}\right) \geq & \left\lceil\frac{f\left(v_{1}\right)+f\left(v_{1}^{\prime}\right)}{2}\right\rceil+ \\
& d\left(v_{1}, v_{1}^{\prime}\right) \\
\geq & \left\lceil\frac{2+3}{2}\right\rceil+1=4 \\
\geq & n
\end{aligned}
$$

From all the above cases it follows that $f$ satisfies Radio mean condition for all pairs of vertices of $T\left(P_{n}\right), \mathrm{n}=2$, 3,4 and the largest integer utilized in this labeling is $\mathrm{n}$ and so $r m n(f)=\mathrm{n}$ and $f$ is a graceful radio mean labeling. B. Case II: $n=5,6,7$

\section{Theorem: III-B.1}

The total graph $T\left(P_{n}\right)$ of path $P_{n}, n=5,6,7$ admits Radio mean labeling with $\operatorname{rmn}\left(T\left(P_{n}\right)\right)=3 n-6$.

Proof. Define $f: V\left(T\left(P_{n}\right)\right) \rightarrow \mathbb{Z}^{+}$using indices $d_{0}=n, d_{1}=1, d_{2}=3$ as follows:

$$
\begin{aligned}
f\left(v_{1}\right) & =n-3, \\
f\left(v_{2}\right) & =3 n-7, \\
f\left(v_{i}\right) & =n+2 i-7: 3 \leq i \leq n-1, \\
f\left(v_{n}\right) & =n-4, \\
f\left(v_{1}^{\prime}\right) & =3 n-6, \\
f\left(v_{i}^{\prime}\right) & =n+2 i-6: 2 \leq i \leq n-1 .
\end{aligned}
$$

Clearly, $f$ is an injective function. We can also verify as in earlier case that $f$ satisfies (2) and so it follows that $f$ is a radio mean labeling. Since maximum integer assigned to any vertex under this labeling is $3 n-6, r m n(f)=$ $3 \mathrm{n}-6$. When $\mathrm{n}=5, f$ is a graceful labeling and so $\operatorname{rmn}\left(T\left(P_{n}\right)\right)=3 \mathrm{n}-6, \mathrm{n}=5$. And $r m n\left(T\left(P_{n}\right)\right) \leq 3 \mathrm{n}-6, \mathrm{n}=6$, 7. It is clear from the function $\mathrm{f}$ that any radio mean labeling of $T\left(P_{n}\right), \mathrm{n}=6,7$ whose range consists of only integers greater than $\mathrm{n}-4$ has a span greater than that of $f$. 
Let us now consider any radio mean labeling $h$ of $T\left(P_{n}\right), \mathrm{n}=6,7, h: V\left(T\left(P_{n}\right)\right) \rightarrow\{n-5, n-4, n-3, \cdots\}$. Then it follows from Radio mean condition that any vertices receiving labels

$n-5$ and $n-4$ must be at least 4 distance apart, any vertices receiving labels $n-4$ and $n-3$ must be at least 3 distance apart, any vertices receiving labels $n-3$ and $n-2$ must be at least 2 distance apart and any vertices receiving labels $n-2$ and $n-1$ must be 1 distance apart. It can easily be seen that it is not feasible to label the vertices of $T\left(P_{n}\right)$ using integers from $\mathrm{n}-5$ to $\mathrm{n}-1$ satisfying the above constraints on distance. This indicates that not all of these integers are in the Range of $h$ which in turn says that the maximum integer assigned to any vertex under this labeling, $r m n(h)>3 n-7$. In other words, $r m n(h) \geq 3 n-6$. Thus, we can show that any radio mean labeling of $T\left(P_{n}\right), \mathrm{n}=6,7$ whose range set consists of integers less than $\mathrm{n}-4$ has span greater than or equal to $3 \mathrm{n}-6$. Hence, $\operatorname{rmn}\left(T\left(P_{n}\right)\right)=3 \mathrm{n}-6, \mathrm{n}=6,7$.

Therefore, for the total graph $T\left(P_{n}\right)$ of path $P_{n}, \mathrm{n} \in\{5,6,7\}, \operatorname{rmn}\left(T\left(P_{n}\right)\right)=3 \mathrm{n}-6$.

C. Case III: $n \geq 8$

\section{Theorem: III-C.1}

The total graph $T\left(P_{n}\right), n \in[8,12]$ admits Radio mean labeling with $\operatorname{rmn}\left(T\left(P_{n}\right)\right)=3 n-7$.

Proof. Let $\mathrm{n} \in[8,12]$. Define $f: V\left(T\left(P_{n}\right)\right) \rightarrow \mathbb{Z}^{+}$using indices $d_{0}=n, d_{1}=1, d_{2}=3, d_{3}=5, d_{4}=5$ as follows:

$$
\begin{aligned}
& f\left(v_{1}\right)=n-4, \\
& f\left(v_{i}\right)=3 n+2 i-16: i=2,3,4, \\
& f\left(v_{5}\right)=n-2, \\
& f\left(v_{i}\right)=n+2 i-12: 6 \leq i \leq n-1, \\
& f\left(v_{n}\right)=n-5, \\
& f\left(v_{i}^{\prime}\right)=3 n+2 i-13: i=1,2, \\
& f\left(v_{3}^{\prime}\right)=n-3, \\
& f\left(v_{4}^{\prime}\right)=3 n-7, \\
& f\left(v_{5}^{\prime}\right)=n-1, \\
& f\left(v_{i}^{\prime}\right)=n+2 i-11: 6 \leq i \leq n-1 .
\end{aligned}
$$

Clearly, $f$ is an injective function. We can also verify that $f$ satisfies (2) and so it follows that $f$ is a radio mean labeling. Since maximum integer assigned to any vertex under this labeling is $3 n-7, r m n(f)=3 n-7$. And $r m n(T$ $\left.\left(P_{n}\right)\right) \leq 3 \mathrm{n}-7, \mathrm{n} \in[8,12]$. It is clear from the function $\mathrm{f}$ that any radio mean labeling of $T\left(P_{n}\right), \mathrm{n} \in[8,12]$ whose range consists of only integers greater than $\mathrm{n}-5$ has a span greater than that of $f$. Let us now consider any radio mean labeling $h$ of $T\left(P_{n}\right), \mathrm{n} \in[8,12], h: V\left(T\left(P_{n}\right)\right) \rightarrow\{\mathrm{n}-6, \mathrm{n}-5, \mathrm{n}-4, \cdots\}$. Then it follows from Radio mean condition that any vertices receiving labels $n-6$ and $n-5$ must be at least 5 distance apart, any vertices receiving labels $n-5$ and $n-4$ must be at least 4 distance apart, any vertices receiving labels $n-4$ and $n-3$ must be at least 3 distance apart and any vertices receiving labels $n-3$ and $n-2$ must be 2 distance apart. It can easily be seen that it is not feasible to label the vertices of $T\left(P_{n}\right)$ using integers $n-6$ to $n-2$ satisfying the above constraints on distance. This indicates that not all of these integers in the range of $h$ and hence the maximum integer assigned to any vertex under this labeling, $r m n(h)>3 n-8$. In other words, $r m n(h) \geq 3 n-7$. Thus, we can show that any radio mean labeling of $T\left(P_{n}\right), \mathrm{n} \in[8,12]$ whose range set consists of integers less than $\mathrm{n}-5$ has span greater than or equal to $3 \mathrm{n}-7$. Hence, $\operatorname{rmn}\left(T\left(P_{n}\right)\right)=3 \mathrm{n}-7, \mathrm{n} \in[8,12]$.

\section{Lemma: III-C.1}

Suppose $n$ is any integer which belongs to an interval of the form : $\left[8+S_{k}, 12+S_{k}+k\right]$ where $S_{k}=5+6+7+\cdots$ $+(5+k-1)$ and $k=1,2,3, \cdots$. Then there exists a Radio mean labeling of $T\left(P_{n}\right), n \geq 13$ with radio mean number $3 n-k-7$.

Proof. Let us define a function $f: V\left(T\left(P_{n}\right)\right) \rightarrow \mathbb{Z}^{+}$using indices $\mathrm{d}_{0}, \mathrm{~d}_{1}, \mathrm{~d}_{2}, \cdots$ where

(i) $d_{0}=n$,

(ii) $d_{1}=1$,

(iii) $d_{2}=n-\left[f\left(v_{d_{1}}\right)+1\right]$,

(iv) $d_{i}=d_{i-1}+n-\left[f\left(v_{d_{i-1}}^{\prime}\right)+1\right]$, for $i$ is odd, $i \leq k+4$,

(v) $d_{i}=d_{i-1}+n-\left[f\left(v_{d_{i-1}}\right)+1\right]-1$, for $i$ is even, $i \leq k+4$.

The function $f$ is given by 


$$
\begin{array}{rrl}
\text { (i) } & f\left(v_{n}\right)= & f\left(v_{d_{0}}\right)=n-k-5 \\
\text { (ii) } & f\left(v_{1}\right)= & f\left(v_{n}\right)+1=n-k-4 \\
\text { (iii) } & f\left(v_{d_{i}}\right)= & f\left(v_{d_{1}}\right)+i-1=n-k+i-5 \\
& & : \text { if } i \text { is odd }, i \leq k+4 \\
\text { (iv) } & f\left(v_{d_{i}}^{\prime}\right)=\quad & f\left(v_{d_{1}}\right)+i-1=n-k+i-5 \\
& & \text { : if } i \text { is even }, i \leq k+4
\end{array}
$$

$(v) \quad$ if $\mathrm{k}+4$ is odd:

$$
\begin{array}{ll}
f\left(v_{i}^{\prime}\right)=\quad & f\left(v_{d_{k+3}}^{\prime}\right)+2\left(i-d_{k+3}\right) \text { where } \\
& d_{k+3}+1 \leq i \leq n-1 \\
f\left(v_{i}\right)=\quad & f\left(v_{d_{k+4}}\right)+2\left(i-d_{k+4}\right) \text { where } \\
& d_{k+4}+1 \leq i \leq n-1
\end{array}
$$

if $\mathrm{k}+4$ is even:

$$
\begin{array}{rll} 
& f\left(v_{i}^{\prime}\right)=\quad & f\left(v_{d_{k+4}}^{\prime}\right)+2\left(i-d_{k+4}\right) \text { where } \\
& & d_{k+4}+1 \leq i \leq n-1 \\
& f\left(v_{i}\right)=\quad & f\left(v_{d_{k+3}}\right)+2\left(i-d_{k+3}\right) \text { where } \\
& & d_{k+3}+1 \leq i \leq n-1 \\
(v i) \quad f\left(v_{i}^{\prime}\right)=\quad & f\left(v_{n-1}^{\prime}\right)+2 i: 1 \leq i<d_{2} \\
\text { (vii }) \quad f\left(v_{i}\right)=\quad & f\left(v_{n-1}\right)+2(i-1): 2 \leq i<d_{3} \\
\text { (viii }) \quad f\left(v_{i}^{\prime}\right)=\quad & f\left(v_{\left(\left(d_{l}\right)-1\right)}^{\prime}\right)+2\left(i-d_{l}\right) \text { where } \\
& & d_{l}<i<d_{l+2}, l=2,4, \cdots, l \leq k+4 \\
(\text { ix }) \quad f\left(v_{i}\right)=\quad & f\left(v_{\left(\left(d_{l}\right)-1\right)}\right)+2\left(i-d_{l}\right) \text { where } \\
& & d_{l}<i<d_{l+2}, l=3,5, \cdots, l \leq k+4
\end{array}
$$

Clearly, $f$ is an injective function. We can also verify that $f$ satisfies (2) and so it follows that $f$ satisfies Radio mean condition for all pairs of vertices of $T\left(P_{n}\right)$ where $\mathrm{n} \in\left[8+\mathrm{S}_{\mathrm{k}}, 12+\mathrm{S}_{\mathrm{k}}+\mathrm{k}\right]$ where $\mathrm{S}_{\mathrm{k}}=5+6+7+\cdots+(5+$ $\mathrm{k}-1$ ) and $\mathrm{k}=1,2,3, \cdots$. The largest integer used under this mapping is $3 \mathrm{n}-\mathrm{k}-7$. Hence the radio mean number of $f$, and the $r m n(f)=3 \mathrm{n}-\mathrm{k}-7$.

\section{Theorem: III-C.2}

For $T\left(P_{n}\right), n \in\left[8+S_{k}, 12+S_{k}+k\right]$ where $S_{k}=5+6+\cdots+(5+k-1)$ and $k=1,2,3, \cdots$ as in previous lemma, the radio mean number $r m n\left(T\left(P_{n}\right)\right) s 3 n-k-7$.

Proof: Proof follows from the preceding lemma.

Fig:2 Total graph of Path on 8 vertices

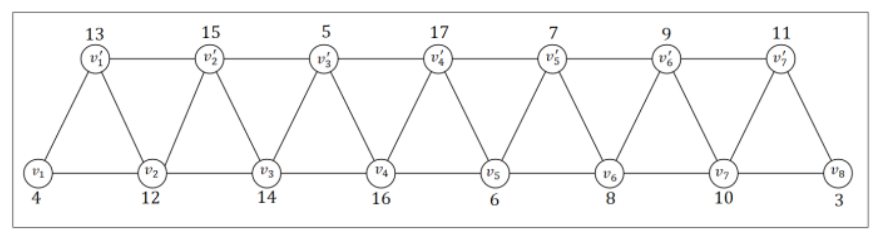

\section{CONCLUSION}

In this paper, authors have obtained Radio mean labelings of Path graph and its total graph with minimum span. The radio mean number of $P_{n}$ is given by

$$
\operatorname{rmn}\left(P_{n}\right)= \begin{cases}n & \text { if } n=2,3, \\ 2 n-4 & \text { if } n=4,5,6, \\ 2 n-k-4 & \text { if } n \in\left[4+S_{k}, 6+S_{k}+k\right], \\ & k \in \mathbb{N}, \\ & S_{k}=3+4+\cdots+(3+k-1) .\end{cases}
$$

And the radio mean number of $T\left(P_{n}\right)$ is given by 
$\operatorname{rmn}\left(\mathcal{T}\left(P_{n}\right)\right)= \begin{cases}2 n-1 & \text { if } n=2,3,4, \\ 3 n-6 & \text { if } n=6,7, \\ 3 n-7 & \text { if } n=8,9,10,11,12, \\ 3 n-k-7 & \text { if } n \in\left[8+S_{k}, 12+S_{k}+k\right], \\ & k \in \mathbb{N}, \\ & S_{k}=5+6+\cdots+(5+k-1)\end{cases}$

\section{REFERENCES}

1. Chartrand, Gary, Cooroo Egan and Ping Zhang. How to Label a Graph. Springer International Publishing, 2019.

2. Gallian, Joseph A. "A dynamic survey of graph labeling." The Electronic Journal of Combinatorics 17 (2014) : $60-62$.

3. Lavanya Y. and K. N. Meera. "Radio Mean Graceful Graphs." Journal of Physics: Conference Series. Vol. 1172. No. 1. IOP Publishing, 2019.

4. K. N. Meera, "Radio Geometric graceful graph", IOP conference series : Material Science and Engineering, 577(2019), 012167.

5. Ponraj, R., S. Sathish Narayanan and R. Kala. "Radio mean labeling of a graph." AKCE International Journal of Graphs and Combinatorics 12.2 - 3(2015) : 224 - 228.

6. Ponraj, R., and S. Sathish Narayanan "On radio mean number of some graphs." International journal of Mathematical Combinatorics 3 (2014) : 41.

7. Ponraj, R., S. Sathish Narayanan and R. Kala. "Radio mean number of some wheel related graphs." Jordan Journal of Mathematics and Statistics (JJMS) 7 .4(2014) : 273 - 286.

8. Ponraj, R., S. Sathish Narayanan and R. Kala. "Radio mean number of some subdivision graphs." Jordan Journal of Mathematics and Statistics (JJMS) 9 .1(2016) : 45 - 64.

9. Ponraj, R. and S. Sathish Narayanan "Radio Mean Number of Certain Graphs." International Journal of Mathematical Combinatorics 2 (2016) : 51.

10. Radha Ramani Vanam and K. N. Meera. "Radio degree of a graph." AIP Conference Proceedings. Vol. 1952. No. 1. AIP Publishing LLC, 2018.

11. Radha Ramani Vanam, K. N. Meera and Dhanyashree. "Improved bounds on the Radio degree of a cycle." IOP Conference Series: Materials Science and Engineering. Vol. 577. No. 1. IOP Publishing, 2019.

12. Raj, C. DAVID, M. Deva Saroja and Brindha Mary VT. "RADIO MEAN LABELING ON DEGREE SPLITTING OF GRAPHS." The International journal of analytical and experimental modal analysis

13. Smitha, KM Baby and K. Thirusangu. "ON THE RADIO MEAN NUMBER OF CORONA GRAPHS." International Journal of Pure and Applied Mathematics 118 .10(2018) : 223 233.

14. Sunitha, K., C. David Raj and A. Subramanian. "Radio mean labeling of Path and Cycle related graphs." Global Journal of Mathematical Sciences: Theory and Practical 9 .3(2017) : $337-345$.

15. Van den Heuvel, Jan, Robert A. Leese and Mark A. Shepherd. "Graph labeling and radio channel assignment." Journal of Graph Theory 29.4(1998) : 263 - 283.

16. West, Douglas Brent. Introduction to graph theory. Vol. 2. Upper Saddle River, NJ: Prentice hall, 1996.

17. Qi, Hengxiao, et al. "Radio Labeling for Strong Product $K_{3} \otimes P_{n}$." IEEE Access 8 (2020): 109801-109806.

18. Badr, Elsayed, et al. "An Integer Linear Programming Model for Solving Radio Mean Labeling Problem.” IEEE Access 8 (2020): 162343-162349. 